\title{
Design of Security Monitoring System for Geological Data Library of Hainan Province
}

\author{
Min Sun ${ }^{1, a}$ and Binwen Huang ${ }^{2, b}$ \\ 1,2 Information Technology Department, Hainan Vocational College of Political Science and Law, \\ Haikou, Hainan, 571100, China \\ email: ${ }^{3} 30516607 @ q q . c o m,{ }^{b} 64471362 @ q q . c o m$
}

Keywords: Geological data; Security monitoring; Ethernet

\begin{abstract}
Geological Data Library is an important component of Land and Environmental Resources Department of Hainan Province, which is responsible for the collection and management of Hainan province's regional geological data, such as geological survey data, solid mineral geology data, petroleum geology data, marine geology data, physical and chemical exploration data, hydrogeology data, engineering geology data and geological research data etc. Firstly, the author analyzes the hidden problems of Hainan Geological Data Library, and then simply introduces the characteristics of distributed measurement and control system, finally elaborates the design of security monitoring system of Geological Data Library.
\end{abstract}

\section{Situation Analysis of Geological Data Library}

Protection degree of collection geology data is low. In recent years, each city geological team has transferred less information to the Provincial Geological Data Library. Although the received data of city geological team contains a certain proportion Class B data that needn't to be transmitted to Provincial Geological Data Library, the data gap is not so large. The number of data that is transmitted from individual city geological team to the Provincial Geological Data Library is zero for three consecutive years. Submission situation of marine geology data that should be directly submitted to Provincial Geological Data Library is not ideal; some provincial large special work data hasn't been submitted. Library users complain no new substantial material in multiple user survey. Many issues are reflected from the survey, for example, poor quality in few submission data, and inconsistent contents between electronic documents and paper information.

The progress of comprehensive research and excavation work of collection geology data is slow. The workload of provincial library staff was added more than doubled due to parallel double-track running mode between the paper archives of traditional manual management and digital modern archives. In addition, the constraints of the traditional thinking mode, low initiative on the development of collection geology data and weak awareness in turning geology data into service products also affected depth and breadth of service work.

Storage conditions of collection data and basic services work needs to be strengthened. As data processing conditions cannot meet the work requirements in Provincial Library, storage conditions improvement must be on the agenda. Due to the lack of management and top-level design of business technology of national geological data, each test paper referred to inconsistencies, divided in different security classification, different cleanup scales, different classification methods, non-standard acceptance of digital geological data, Repeated low-level information system construction, etc.

\section{The Characteristics of Distributed Measurement and Control System Based on Ethernet}

Ethernet distributed measure and control system complies with TCP/IP protocol and uses Ethernet/Internet as the command and data transmission network. Its structure is no longer like the traditional three-tier structure of the fieldbus measurement and control network: communication computer - fieldbus - measurement and control equipment; but two-tier structure: management 
network - measurement and control network. Its characteristics are listed as follows:

Real-Time. Real-time requirement of distributed measurement and control system is very strict, it requires the system to reliably respond within the specified time. Theoretically Ethernet is not a real-time system, which is the Achilles heel for automatic control. However, in actual use, take $10 \mathrm{Mbps}$ bandwidth as an example, if the transmission data volume is not a large number of video graphics data like in OA environment, the actual collision probability is very low. Even a collision, it can still spread the data very quickly to each other, not to mention the recent growing popularity of switching HUB, you can set the elasticity 10M / 100M dedicated bandwidth to the user. It's like on the highway, it not only has more than 10 lanes for choosing to speed up, but you can specify a dedicated lane to enjoy the president's tour of inspection and unimpeded courtesy.

Reliability. Ethernet cable from the coaxial cable, to twisted pair (UTP), until the optical fiber. Especially fiber-optic network has been gradually extended from telecom's backbone network to the terminal device, it stressed similar RJ-45 phone Jack flexible fiber-optic connectors and high flexibility fiber and low-cost, the site is easy for construction. The optical fiber is not affected by noise interference, it is suitable for real-time monitoring in the high noise environment of the scene. On the other hand, a standard SNMP network management tools can be used to effectively manage the network.

Compatability. TCP / IP communication protocol is an international common standard, both Intranet and Internet are using this standard, various brands of computers and field devices have the same communications infrastructure. Field device which has a TCP/IP interface can connect directly to Internet, it realizes remote control or remote diagnostics function.

Resource Sharing. Because communication packet matches TCP/IP standard, it can pass through the INTERNET switch or router. TCP/IP communication protocol is a global uniform standard, easily linked into large control network without geographical restrictions. Ethernet measurement and control uses Ethernet (Internet) which is the most extensive resources, it make Ethernet measurement and control system has the infinite region and number of measure and control nodes.

Cost Effective. There are the largest vendors and cheapest price of hardware and software for Ethernet. If we use dedicated Field Bus as automation network architecture, all the hardware procurement, wiring construction, software development and maintenance etc. are subject to this architecture, the cost is high.

Future Trend. Development trend of Internet/Intranet has being from OA ->FA ->BA, right now, the trend is actively extended to HA home automation field. One of the most difficult is wiring, so telephone line Home PNA and wireless RF etc. wiring technology are developing now. And due to the vast number of market forces, these techniques is upgrading rapidly, the cost is relative decline, the future is bound in the reverse drive to BA, FA, OA field.

\section{Implementation of Security Monitoring System of Geological Data Library}

Security monitoring system of Hainan Geological Library is made up of various city geological data monitoring subsystem, private network of provincial geological resources, provincial geological data library security monitoring host. Its function is to monitor, upload information and alarm automatically according to the safety of various City Geological Data Library (such as high temperature, humidity, drastic changes in temperature and humidity, power outages and water in typhoon season etc.). In addition, it also can receive the operation control signal of the upper machine, such as alarm threshold, sampling time, reset, etc. Due to private IP network of Hainan Province Geological Resource has been constructed, and combining the characteristics of the Ethernet control system, the distributed Ethernet control system is adopted to build.

Each city geological database monitoring subsystem is as a generated point of information flow in, its essence is to complete the security data collection for various cities geological data library, send messages to PC of city own database monitoring center through private IP network after a certain processing, and the city local geological information will be reported to the Provincial Geological Data Library using geological resources dedicated network by monitoring center host. 
That is, each city geological database monitoring and controlling system is a distributed Ethernet control system.

Ideas of Security Monitoring System. The security monitoring system which is integrated with computer technology, communication technology, network technology, geological information management and services, and many other environmental monitoring systems features. The system also can collect and monitor environmental data of Geological Data Library. The design not only need to achieve the purpose of environmental data acquisition and monitoring, but also meet the requirements of data transmission standards which specified in the Land and Environmental Resources Department, in addition, real-time data transmission, reliability, safety and other requirements should be considered in the design.

The system structure adopts modular design, it mainly contains two parts: monitor and security mornitoring operating management software in Geological Data Library. The monitor which can collect data and alarm according to requirement, and upload the data via Ethernet to the management machine, and can also receive parameter settings from security monitoring and management software via Ethernet. Since the design of monitor is independence, the security monitoring system upgrades, debugging expansion and system integration has been guaranteed.

Functions of Security Monitoring System. The system use Ethernet measurement and control technology to implement the function of data collection and environment monitoring of Geological Data Library, it includes timely alarm, timer, or real-time data acquisition, switch control and detection. Provincial Geological Data Library can easily view all cities library security situation, so as to ensure reliable transmission of information between all cities data library and the Provincial Data Geological Library, and reduce the expenditures of manpower and financial resources of the Data Library to further enhance the geological archives of the management level, reducing expenditure of human and financial resources, and Geological Data Library of the management level has been further improved. Through the establishment of the system to achieve the following objectives:

Environmental data collection and monitoring: Timing or real-time data collection for each environment, as required, upload the own monitor of Geological Data Library; Determine whether the various environmental parameters exceed the alarm threshold, sent alarm information to monitoring center immediately if there is an exception; receive and execute control commands in real time, which form PC of Provincial Geological Data Library monitoring center.

Provincial geological data library security monitoring host: On the one hand, it has the function of two-way communication with the monitoring device, it can real time or timing receive environment data and alarm information uploaded from City Geological Data Library monitor, record control orders in the database for queries and transmit control command. The data is uploaded to PC of the Provincial Geological Data Library monitoring center right away. On the other hand, it provides the user with a visual interface which can display the total appearance of monitoring system in real time, library equipment operating status, alarm information and statistical results of various environmental data, so that the staff of geological data library don't need go out and can intuitively understand the local library environment.

The System Structure. To achieve the system function as the starting point, after considering and analyzing security systems application environment of Geological Data Library, related hardware and software technical level, the construction cost, operability, and many other conditions, the structure of the system is determined later. The overall system implementation illustrated in Fig. 1 : 


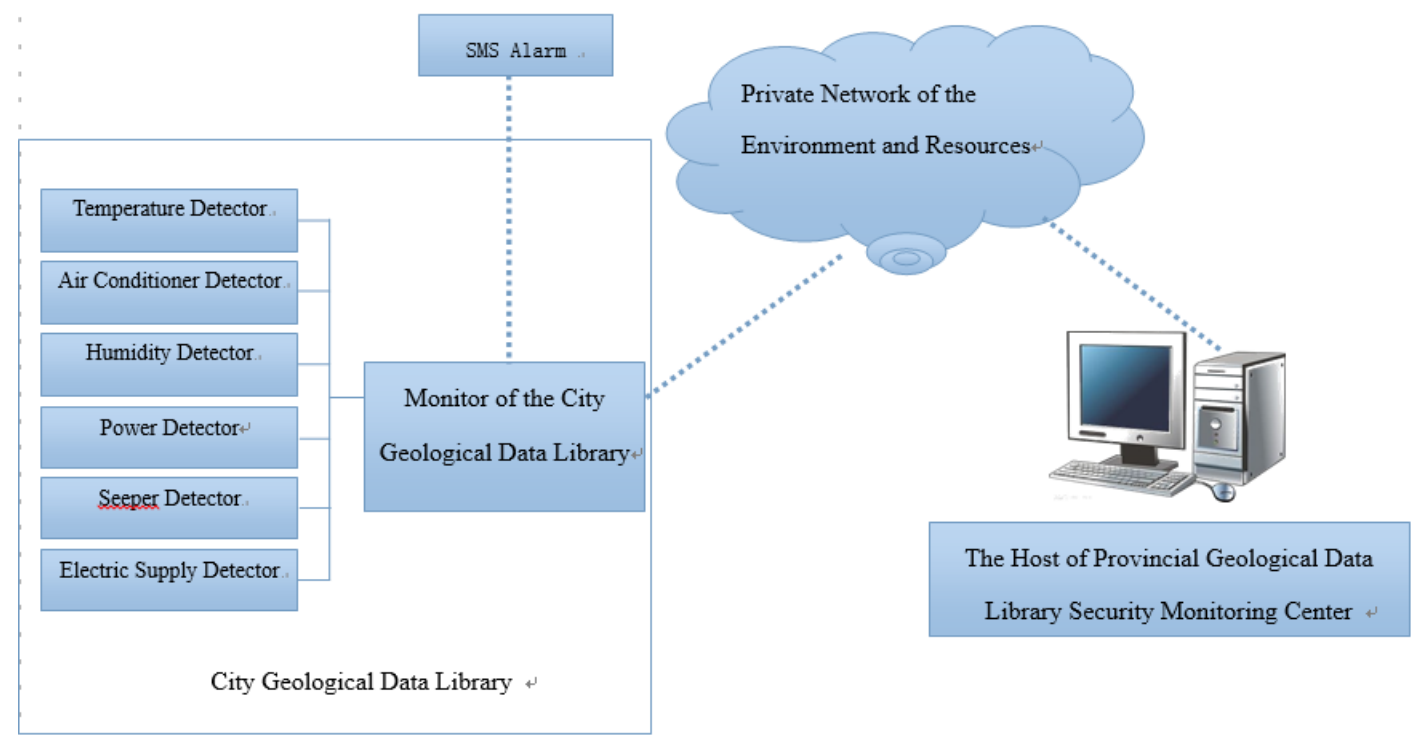

Fig.1. The structure of the system

Function of environment detectors: Temperature detector, which can measure automatically the temperature of various City Geological Data Library or Provincial Geological Data Library, and transmit the temperature data to the monitor of appropriate library.

Humidity detector, which can measure automatically air humidity of the various City Geological Data Library or Provincial Geological Data Library, and transmit the humidity data to the monitor of appropriate library.

Air conditioner detector, which monitor air conditioner is woking or not, and transmit the signal(on/off) to the monitor of appropriate library.

Seeper detector, which can measure whether there is water under the floor, and the presence or absence of water data would be transmitted to the the monitor of appropriate data library.

Power detector, which can monitor output of AC voltage of UPS, load current, transmit the data to the monitor of appropriate data library, and automatic timing settings for UPS battery discharge.

Electric supply detector, which can monitor automatically monitoring power supply situation of City Geological Data Library or Provincial Geological Data Library, record power outages,and calculate UPS discharge time.

Distribution of detectors of City Geological Data Library were shown in Figure 2.

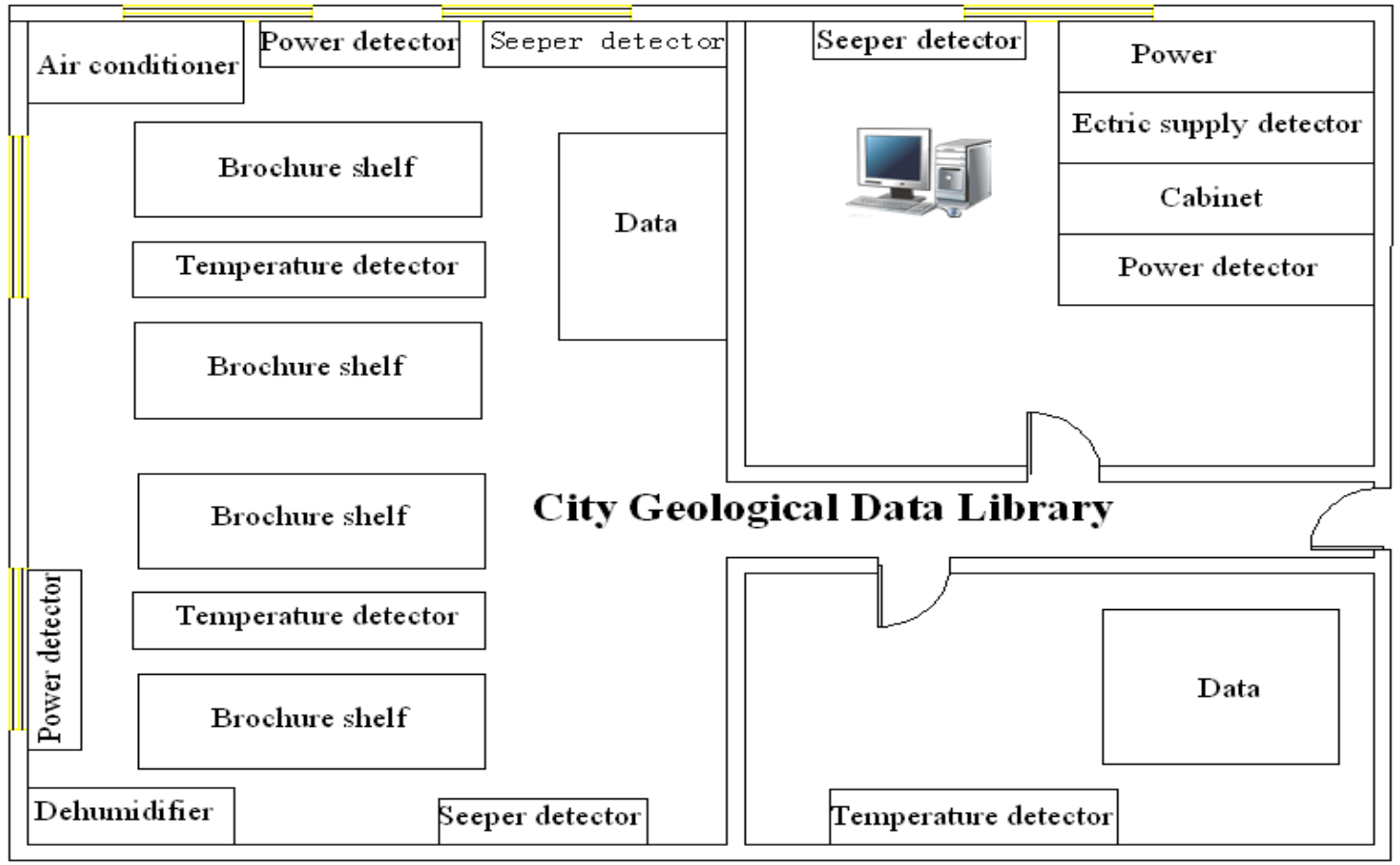

Fig.2 The City Geological Data Library 
City Geological Data Library monitoring. City Geological Data Library monitor using a fixed IP address connect private IP network of Province Environmental Resource, and connect temperature detectors, humidity detectors, air conditioner detectors, seeper detectors, power detectors, and electric supply detectors. It's mainly in a listening state when it works.

Provincial Geological Data Library security monitoring host. Provincial Geological Data Library security monitoring host, including the hosts of City Geological Data Library and Provincial Geological Data Library. Monitoring center host has the ability to connect Ethernet, it is a PC or server which can manage the entire system or subsystem. Provincial monitoring center host can receive all information from the control nodes (the city geological team database), then optimize the process, issuing various control commands, statistics and analysis, and establish a database.

Under normal circumstances, all cities geological team receives environmental monitoring data from its own Geological Data Library, and then forwards to the provincial monitoring center, on the one hand, it is responsible for the security of Provincial Geological Data Library, and on the other hand, we can understand the security situation of City Geological Data Library by provincial security monitoring host. In addition, the host can also sent some control commands to all city geological teams.

\section{Conclusions}

Since security monitoring system of Hainan Geological Data Library is accepted and used, the system is running well, it have met the requirements of Land and Environmental Resources Department of Hainan Province, and provides a reference to further management. New management means combined with constantly improvement of the management system, adhere to the "people-oriented", it let the management work of geological data more humane, and it fully mobilizes the enthusiasm of the city geological staff, improve working enthusiasm and self-consciousness, reduce administrative burden of geological staff, and improve the work efficiency.

\section{References}

[1] National Archives. Archives of temperature and humidity management Interim Provisions.1985

[2] State Department. Geological Data Management Regulations. 2002

[3] Zhang Jin. Thinking of geological archives of information technology. Sichuan file. 2004:20-21

[4] Wang Changli, Roan.Distributed Control System Design and Application Examples. Beijing:Electronic Industry Press. 2005

[5] Pan Shibin. Ethernet gateway design and application of network monitoring and control system [Dissertation]. East China Institute of Technology. 2002

[6] Song Chen. Application Technology of Embedded Control System in Distributed Control Systems. 2010 